\section{Quantum particle filter: a multiple mode method for low delay abrupt pedestrian motion tracking}

\author{
A. Khalili ${ }^{\bowtie}$, A.A. Soliman and M. Asaduzzaman
}

Pedestrian tracking is a key building block in many emerging technologies, and there are many applications that require a very accurate and reliable pedestrian tracking. Two problems, namely the abrupt changes in motion and the delay in the tracking process are addressed in the reported work. The approach seeks to simulate the uncertainty of the position of electrons around the nucleus by propagating particles in areas where the person is more likely to be, depending on the person's possible motions and taking into account various sources of uncertainty. A novel feature of the presented approach is that it can track abrupt motions accurately with a low delay. The robustness of this approach for abrupt motion situations is demonstrated.

Introduction: Pedestrian tracking is a key building block in many applications such as surveillance, abnormal activity detection and dynamic pedestrian avoidance for autonomous vehicles. Pedestrian tracking is very challenging because people often change their motion abruptly, which causes the failure of most tracking methods.

In practice, a prior knowledge about object motions is assumed. Motion models such as constant velocity $(\mathrm{CV})$ and constant acceleration (CA) are used to predict object motion [1]. However, these models are too general to model various types of motions such as abrupt changes in speed or in direction, which leads to degradation of tacking accuracy. One solution to the abrupt motion problem is to search the whole state space to cope with motion uncertainty; however, this requires a high computational cost. Kristan et al. [2] proposed a two-stage dynamic model: a liberal model and a conservative model to improve the accuracy of the particle filter. However, this method fails when the frequency of abrupt motions is high. Other researchers [3, 4] proposed methods that use learned motion models; however, the main limitation of these methods is that they are limited to motions which they are trained for.

To cope with various pedestrian motions, some authors have proposed using the interacting multiple mode (IMM) method [5], which is based on multiple trackers, each of them trying to track different motion models. IMM performance depends on how well the models match with the actual dynamics; furthermore, if there are many models used, the performance will degrade rapidly. IMM also suffers from the mode estimation delay problem which is the time of probability convergence to the true model. This increases the error and causes serious limitations in some applications such as pedestrian avoidance for autonomous vehicles. Madrigal and Hayet [6] used the interacting multiple mode particle filter (IMMPF) for pedestrian tracking, and although the IMMPF could be used for nonlinear and non-Gaussian estimation problems it is not computationally feasible and it has the same previously mentioned limitations of the IMM method.

Baxter et al. [7] extended the Kalman filter to adaptively combine motion information with a prior belief about where the person will go based on where they are currently looking. They showed that the proposed tracker outperforms the Kalman filter for sudden motion changes.

None of the existing methods fully use the available prior information about a pedestrian's possible motions to improve the tracking. In this Letter we use this information to cope with abrupt motions and to reduce the tracking delay. The first contribution of this Letter is the proposition of a pedestrian tracking method that outperforms existing tracking methods when there are abrupt changes in speed or in direction. The second contribution is the reduction of the tracking delay in comparison with other tracking methods.

Quantum particle filter $(Q P F)$ : Pedestrian movement is generally nonlinear. The particle filter [8] is essential to resolve estimation problems in the nonlinear and/or non-Gaussian case; however, this method has serious limitations when there are abrupt motions. To address these limitations, we seek solutions that can quickly guide the algorithm to areas where the person is more likely to be.

The approach tries to simulate the uncertainty of the position of electrons around the nucleus by propagating particles in areas where the person is more likely to be. The proposed method uses the same structure of the particle filter, except for the prediction step. We assumed that the person could abruptly change direction by any angle. We also assumed that at any time instance the person could switch to any of these three main modes: the stop mode, the walking mode and the running mode. Various speeds inside each mode are taken into account by determining the variance of each mode. One more mode, called the current mode, is added to enforce the current dynamic. The basic idea of the proposed method is to place the particles in areas that correspond to the person's possible motion if the person makes abrupt changes in speed or in direction. The particles are propagated according to all these modes simultaneously to cope with any abrupt changes. For example, if the person wants to suddenly stop, walk, run or change direction, the particles will already exist at these possible positions according to the proposed prediction model, which will solve the abrupt motion problem and reduce the tracking delay. To enforce the current dynamics, more particles are propagated using the current speed and direction. However, when the uncertainty of the speed estimation increases rapidly, the particles are equally distributed among all modes to cope with any abrupt changes in speed as illustrated in Fig. $1 b$. The variance of each mode in Fig. 1 is reduced initially for illustration purpose.

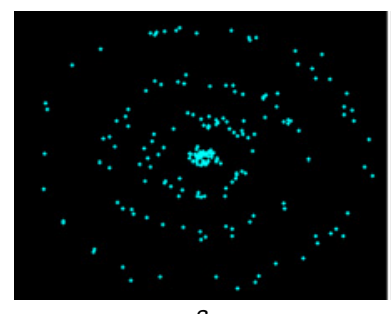

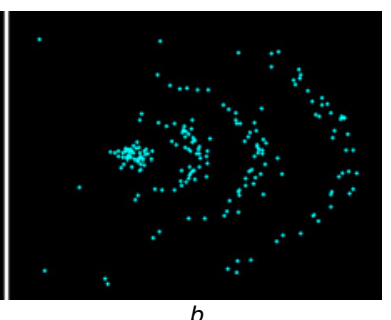

Fig. 1 Quantum particle filter

a Particles are propagated in all directions

$b$ Particles are propagated in current direction

When the person stops suddenly or when the uncertainty of the direction estimation increases rapidly due to a possible hard turn for example, the angular variance of the particles will increase to cover all directions and the particles will be equally distributed in all directions to cope with any abrupt changes in direction as illustrated in Fig. $1 a$.

The particles are propagated according to the following equations:

$$
\begin{aligned}
X_{i}= & R_{i} \cos (\mathbf{A N G L E S})+\mathbf{S V}+x_{\text {centre }} \\
Y_{i}= & R_{i} \sin (\mathbf{A N G L E S})+\mathbf{S V}+y_{\text {centre }} \\
& \mathbf{A N G L E S ~} \sim N\left(\mu_{\text {angle }}, \sigma_{\text {angle }}\right) \\
& \mathbf{S V} \sim N\left(0, \sigma_{\mathrm{pos}}\right)
\end{aligned}
$$

ANGLES is a vector representing a set of angles sampled from a Gaussian distribution with mean and variance $\mu_{\text {angle }}$ and $\sigma_{\text {angle }}$, respectively. $\mathbf{S V}$ is a vector sampled from a Gaussian distribution representing the uncertainty of the positions of particles with $\sigma_{\text {pos }}$ variance. $R_{i}$ represents the distance between the current position to the $i$ th mode, and is related to the transitional speed of each mode $S_{i}=R_{i}$ /(sampling interval). $x_{\text {centre }}$ and $y_{\text {centre }}$ represent the current position, $X_{i}$ and $Y_{i}$ represent the positions of the particles; they either form full circles or arcs depending on $\sigma_{\text {angle }}$ taking into account that the modes with larger $R_{i}$ should have more particles to cover the larger area that they have. The quantum particle filter algorithm is given by Algorithm 1 .

\footnotetext{
Algorithm 1: Quantum particle filter algorithm

1. Prediction:

A. If the uncertainty of the speed estimation increases rapidly, propagate the particles equally among all modes.

B. Else, enforce particles in the current speed.

C. If the uncertainty of the direction estimation increases rapidly, propagate the particles equally among all directions.

D. Else, enforce particles in the current direction.

E. Propagate the particles according to (1) and (2).

2. Measurement update (compute the importance weights)

3. Resample (replace unlikely samples by the more likely ones)

4. Iterate from step 1.
} 
One limitation of the proposed method is that the resampling should be more frequent to cope with the large number of low weight particles which will increase the computational cost.

Simulation results: Computer simulations were performed to evaluate the proposed tracking method in abrupt motion situations. The person is stopping from time step 0 to 10 , then starts walking at $1 \mathrm{~m} / \mathrm{s}$ from time step 10 to 30 , then turns $90^{\circ}$ and continues to walk until time step 50, then stops from time step 50 to 60 , after that starts running at $3.3 \mathrm{~m} / \mathrm{s}$ from time step 60 to 80 , and finally stops from time step 80 to 90. Gaussian noise was added to the true positions with $(\mu=0, \sigma=$ $0.01)$, the averages $S_{1}$ and $S_{2}$ were approximated to 1.3 and $3.5 \mathrm{~m} / \mathrm{s}$. For the comparative evaluation, we compared the proposed method with two different tracking methods, the multiple mode particle filter (MMPF) and the interactive multiple mode (IMM) with the following two motion models: $\mathrm{CV}$ and CA. The MMPF is similar to the IMMPF but without the interaction step to reduce the mode decision delay, where each set of particles is propagated according to one motion model.

The results were obtained from 100 Monte Carlo runs. Fig. 2 shows that the position estimation of the QPF outperformed the MMPF and the IMM. The performance of the IMM and the MMPF decreases when there are severe abrupt motions. Although the MMPF shows a lower positioning error than the IMM, it frequently fails to track the person when the person abruptly changes position.

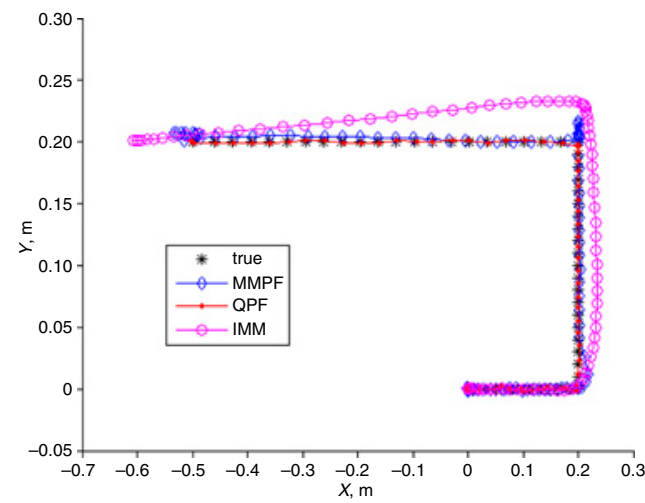

Fig. 2 Trajectory tracking

Fig. 3 shows the positioning error for the three methods; QPF shows the lowest positioning error, IMM positioning error starts at time step 30 (at the turn) due to the linearity of the IMM, then the error increases, largely starting from time step 60 (at the beginning of the abrupt motion), this is mainly due to the mode decision delay. The MMPF positioning error starts at time step 60 and continues to increase until time step 90 , this being mainly due to failure to track the abrupt motion.

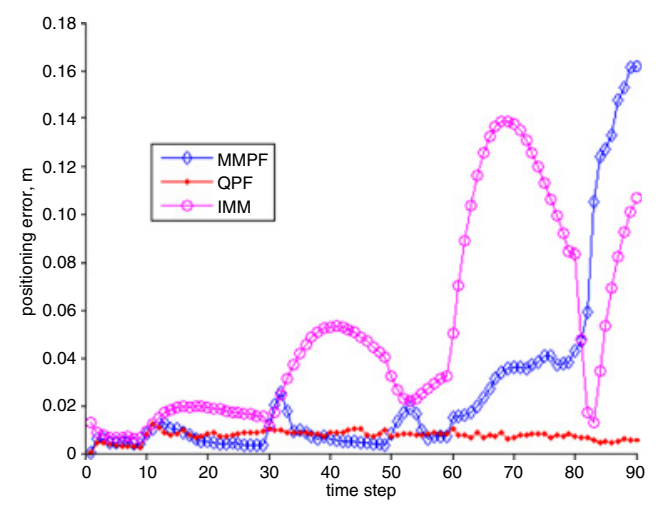

Fig. 3 Positioning error

Fig. 4 shows the tracking delay using the three methods; QPF shows the lowest tracking delay, IMM shows the highest tracking delay, particularly at time step 60 when the abrupt motion started, this being mainly due to the mode decision delay. The MMPF shows a lower delay than the IMM because of the absence of the mode decision delay and the multimodality of the particle filter. The analysis above shows that the QPF has greater robustness, accuracy and lower delay in tracking abrupt motions. The simulation also indicated that the QPF can successfully cope with large motion uncertainty.

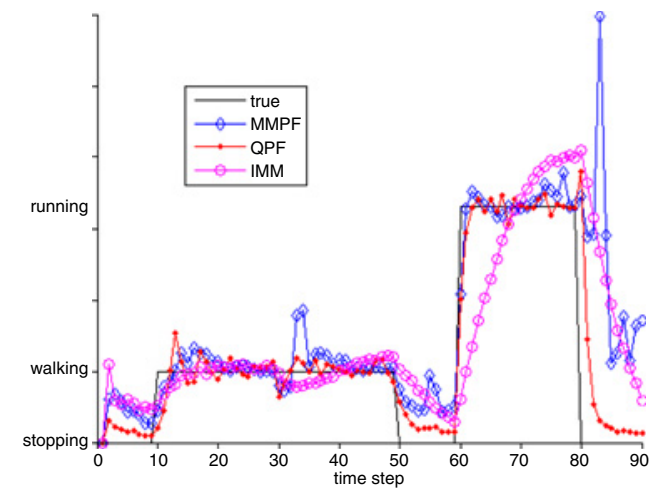

Fig. 4 Tracking delay

Conclusion: This Letter has presented a novel approach for pedestrian tracking that can track abrupt motions accurately with a low delay by propagating particles in areas where the person is more likely to be. We have demonstrated through simulation that the QPF outperformed both the IMM and the MMPF methods. We are planning to extend this work to adapt to other types of objects such as cars. We believe that such an approach holds great promise for accurate and reliable tracking in challenging environments.

(C) The Institution of Engineering and Technology 2015

Submitted: 15 April 2015

doi: $10.1049 /$ el.2015.1013

One or more of the Figures in this Letter are available in colour online.

A. Khalili, A.A. Soliman and M. Asaduzzaman (Faculty of Computing, Engineering and Technology, Staffordshire University, Stoke on Trent, United Kingdom)

凶 E-mail: k026252e@student.staffs.ac.uk

\section{References}

1 Li, X.-R., and Jilkov, V.: 'Survey of maneuvering target tracking', IEEE Trans. Aerosp. Electron. Syst., 2003, 39, (4), pp. 1333-1364, doi: 10.1109/TAES.2003.1261132

2 Kristan, M., Kovacic, S., Leonardis, A., and Pers, J.: 'A two-stage dynamic model for visual tracking', IEEE Trans. Syst. Man Cybern. B, Cybernet., 2010, 40, (6), pp. 1505-1520, doi: 10.1109/ TSMCB.2010.2041662

3 Madrigal, F., Rivera, M., and Hayet, J.: 'Learning and regularizing motion models for enhancing particle filter-based target tracking'. PSIVT, Gwangju, Korea, November 2011, pp. 287-298, doi: 10.1007/ 978-3-642-25346-1_26

4 Pavlovic, V., Rehg, J.M., and MacCormick, J.: 'Learning switching linear models of human motion'. Proc. of 13th Annual Conf. on Neural Information Processing Systems, Vancouver, Canada, December 2001, pp. 981-987

5 Blom, H.A.P., and Bar-Shalom, Y.: 'The interacting multiple model algorithm for systems with Markovian switching coefficients', IEEE Trans. Autom. Control, 1988, 33, (8), pp. 780-783, doi: 10.1109/9.1299

6 Madrigal, F., and Hayet, J-B.: 'Evaluation of multiple motion models for multiple pedestrian visual tracking'. 10th IEEE Int. Conf. (AVSS), Krakow, Poland, August 2013, pp. 31-36, doi: 10.1109/ AVSS.2013.6636612

7 Baxter, R., Leach, M.J.V., Mukherjee, S.S., and Robertson, N.M.: ‘An adaptive motion model for person tracking with instantaneous head-pose features', IEEE Signal Process. Lett., 2015, 22, (5), pp. 578-582, doi: 10.1109/LSP.2014.2364458

8 Gordon, N., Salmond, D., and Smith, A.F.M.: 'Novel approach to nonlinear and non-Gaussian Bayesian state estimation'. Proc. F, Radar Signal Process. 1993, 140, (2), pp. 107-113, doi: 10.1109/78.978374 